\section{Anesthetic Management of a Patient With Obstructive Sleep Apnea and Narcolepsy}

(Scr Med 2011;42:102-3)
Ljuba Stojiljkovic, Amber

\section{Zendner}

Department of Anesthesiology, Northwestern University Feinberg School of Medicine, Chicago IL

\section{Correspondence}

Ljuba Stojiljkovic, M.D., Ph.D. Department of Anesthesiology, Northwestern University Feinberg School of Medicine, Chicago IL, USA Email: ssilj@aol.com

Submitted: September 21, 2011

Accepted: September 26, 2011
A 59-year-old male (height $157 \mathrm{~cm}$, weight $93 \mathrm{~kg}$, BMI 37.6) with history of obstructive sleep apnea (OSA) and narcolepsy was scheduled for laparoscopic sigmoid colon resection and left lighted ureteral stent placement for recurrent diverticulitis. The patient was taking both methylphenidate and modafinil for the treatment of narcolepsy, and was on home continuous positive airway pressure (CPAP) machine for OSA. Other medical history was unremarkable. In the pre-operative area the patient consented for a combined general-epidural anesthesia. $\mathrm{He}$ received midazolam 1mg intravenously (IV) and fentanyl $50 m c$ IV prior to placement of thoracic (T9-10) epidural catheter. The patient was taken to the operating room and general anesthesia was induced with propofol 150mg IV and rocuronium 5omg IV. A combined general-epidural anesthesia was maintained with desflurane and $0.25 \% \mathrm{bu}-$ pivacaine infusion at 4-5 $\mathrm{mL} / \mathrm{hr}$ via the epidural catheter. At the conclusion of the procedure, the patient was successfully awakened, extubated and transferred to the postanesthesia care unit (PACU). Of note the patient did not receive any opiates during the procedure. During the two hour PACU stay, he was started on a patient controlled epidural analgesia (PCEA) with background continuous infusion of $4 \mathrm{~mL} / \mathrm{hr}$ of mixture of $0.1 \%$ bupivacaine and $5 \mathrm{mcg} /$ $\mathrm{mL}$ fentanyl, and patient controlled boluses of $4 \mathrm{~mL}$ of the same bupivacaine/fentanyl mixture with 15 min lockout interval. During PACU stay patient did not complain of any pain, and oxygen saturation was maintained above $95 \%$ on room air. Subsequently he was discharged to continuous oxygen saturation-monitored bed, and his home settings of CPAP were used overnight. During first two postoperative days (POD) pain was well controlled with the PCEA and $30 \mathrm{mg}$ of ketorolac every 6 hours IV. On POD\#2 patient returned his bowel function (BF) and his home medications for narcolepsy (modafinil and methylphenidate) were re- started. The epidural catheter was removed and oral acetaminophen-hydrocodone (325mg/10mg) and ibuprofen were given for pain control. He was discharged home on POD\#4 without any complications.

Obstructive sleep apnea (OSA) and narcolepsy are sleep related disorders ${ }^{1}$. OSA is a disorder characterized by cessation of breathing due to obstruction of the upper airway during sleep $^{2}$. Narcolepsy is characterized by recurrent, uncontrollable episodes of sleep, that may be accompanied in more severe cases by cataplexy (loss of muscle tone without loss of consciousness) and sleep paralysis ${ }^{1}$. Underlying pathophysiological mechanism of narcolepsy is loss of orexinergic neurons in the lateral hypothalamus ${ }^{3}$. Orexin (also known as hypocretin) neuronal pathways are important regulators of sleep/wake cycles, as well as energy homeostasis ${ }^{3}$. Orexigenic neurons produce two neuropeptides, orexin A and orexin B from the same precursor, prepro-orexin ${ }^{3}$. Approximately 90\% of patients with narcolepsy have decreased level of orexin A in cerebrospinal fluid (CSF), and a low level of CSF orexin $\mathrm{A}$ is now recognized as one of the diagnostic criteria for narcolepsy3 ${ }^{3}$. Recently, studies have shown that patients with OSA also have lower levels of orexin A as compared to subjects without $\mathrm{OSA}^{4}$. In addition, there is strong evidence that orexinergic neuronal activation plays an essential role in emerging from general anesthesia ${ }^{5}$.

An estimated 23 million Americans are affected by obstructive sleep apnea and 1 in 2000 Americans have symptoms consistent with narcolepsy. The incidence of patients with both narcolepsy and OSA is unknown and our knowledge on anesthetic management for these patients is limited. In addition, both conditions are associated with increased body mass index and obesity which further complicates anesthetic management. 
Patients with combined OSA and narcolepsy represent unique anesthetic challenge due to their increased sensitivity to anesthetic agents, and risks of prolonged emergence due to opioid-induced respiratory depression and stimulant withdrawal hypersomnia.

Our patient was receiving two central nervous system (CNS) stimulants (modafinil and methylphenidate) which had to be withheld in the perioperative period (until return of BF). Methylphenidate is a CNS stimulant which is approved for treatment of attention deficit hyperactivity disorder, as well as narcolepsy. Mechanism of action involves inhibition of monoamine uptake (dopamine and norepinephrine). Modafinil is a relatively new drug that is approved for treatment of narcoplepsy, and is used as a wake-promoting agent in patients with OSA. Mechanism of action has not been completely understood, however, histaminergic system activation via the orexinergic neurons is recently proposed ${ }^{6}$.

Our major concern in this case was that withdrawal of two potent CNS stimulants may prolong emergence from general anesthesia and cause withdrawal hypersomnia and sleep paralysis. Therefore, we used low-soluble volatile anesthetic desflurane, which allowed rapid titration of general anesthesia and did not result in significant accumulation of the anesthetic. In addition, combination of continuous infusion of local anesthetic bupivacaine, through epidural catheter, decreased the use of the volatile anesthetic and systemic opiates via its powerful anesthetic and analgesic effect.
It is widely accepted that OSA patients are very sensitive to respiratory-depressant effects of narcotics, especially during perioperative period. Therefore, our goal was to minimize the use of systemic opiates, while providing effective postoperative pain relief. Combining general and epidural anesthesia was an effective method to decrease the risk of serious postoperative complications related to OSA and narcolepsy such as apnea, hypersomnia, cataplexy and sleep paralysis.

\section{References}

1. Sansa G, Iranzo A, Santamaria J: Obstructive sleep apnea in narcolepsy. Sleep Medicine 2010; 11: 93-95

2. Strollo PJ, Jr., Rogers RM: Obstructive sleep apnea. N Engl J Med 1996; 334: 99-104

3. Sakurai T, Mieda M: Connectomics of orexin-producing neurons: interface of systems of emotion, energy homeostasis and arousal. Trends Pharmacol Sci; 2011; 32: 451-62

4. Busquets X, Barbe F, Barcelo A, de la Pena M, Sigritz N, Mayoralas LR, Ladaria A, Agusti A: Decreased plasma levels of orexin-A in sleep apnea. Respiration 2004; 71: 575-9

5. Kelz MB, Sun Y, Chen J, Cheng Meng Q, Moore JT, Veasey SC, Dixon S, Thornton M, Funato H, Yanagisawa M: An essential role for orexins in emergence from general anesthesia. Proc Natl Acad Sci U S A 2008; 105: 1309-14

6. Ishizuka T, Murotani T, Yamatodani A: Modanifil activates the histaminergic system through the orexinergic neurons. Neurosci Lett 2010; 483: 193-6 